\title{
Benefícios dos Jogos Não-Digitais no Ensino de Computação
}

\author{
Giani Petri ${ }^{1,2}$, Alejandro Calderón ${ }^{3}$, Christiane G. von Wangenheim², \\ Adriano F. Borgatto ${ }^{2}$, Mercedes Ruiz ${ }^{3}$ \\ ${ }^{1}$ Universidade Federal de Santa Maria (UFSM), Santa Maria/RS, Brasil \\ ${ }^{2}$ Departmento de Informática e Estatística (INE), Universidade Federal de Santa Catarina (UFSC), \\ Florianópolis/SC, Brasil \\ 3Universidade de Cádiz (UCA), Puerto Real (Cádiz), Espanha \\ gpetri@inf.ufsm.br, alejandro.calderon@uca.es, c.wangenheim@ufsc.br, \\ adriano.borgatto@ufsc.br, mercedes.ruiz@uca.es
}

\begin{abstract}
Non-digital educational games (board and card games, paper \& pencil, etc.) are also being used for computing education. However, there are few empirical studies providing sound evidence on the benefits of this kind of games. Consequently, a question that arises is to which regard the expected benefits of these games are real. Thus, the objective of this study is to analyse the benefits of non-digital educational games used for computing education in order to evaluate quality in terms of the players' experience and perceived learning adopting the MEEGA+ games' evaluation model. The results of the evaluation, involving 26 case studies and responses from 509 students, provides evidence that these non-digital games contribute to the students' learning, as well as contribute positively to social interaction, relevance, and fun.
\end{abstract}

Resumo. Jogos educacionais não-digitais (jogos de tabuleiro, cartas, lápis \& papel, etc.) também estão sendo usados para o ensino de computação. No entanto, há poucos estudos empíricos que mostram evidências sobre os beneficios deste tipo de jogos. Consequentemente, uma questão em aberto é se os beneficios esperados destes jogos são, de fato, reais. Assim, o objetivo deste trabalho é analisar os benefícios dos jogos educacionais não-digitais usados para o ensino de computação de modo a avaliar a qualidade em termos de experiência dos jogadores e percepção da aprendizagem adotando o modelo de avaliação de jogos MEEGA+. Os resultados da avaliação, envolvendo 26 estudos de caso e respostas de 509 alunos, evidenciam que os jogos não-digitais contribuem para a aprendizagem dos estudantes, além de contribuir positivamente para a interação social, relevância e diversão.

\section{Introdução}

Nos últimos anos, os jogos educacionais têm sido utilizados como estratégia instrucional para o ensino de computação de modo a contribuir para uma aprendizagem mais efetiva [Battistella e Gresse von Wangenheim, 2016a; Souza e França, 2016]. Esses jogos educacionais, usados como uma estratégia instrucional ativa, possuem características similares aos jogos de entretenimento, envolvendo competição e desafios entre os jogadores, sendo organizados por regras e restrições de modo a alcançar um determinado objetivo [Abt, 2002]. No entanto, os objetivos dos jogos educacionais vão além do entretenimento, eles são projetados especificamente para ensinar as pessoas sobre um determinado assunto, expandir conceitos, reforçar o desenvolvimento, ou 
ajudá-los a explorar ou aprender uma habilidade ou uma mudança de atitude [Abt, 2002].

Embora a maioria dos jogos educacionais existentes para o ensino de computação sejam digitais, atualmente, há também uma tendência na adoção de jogos não-digitais (jogos de tabuleiro, cartas, papel \& lápis, etc.) [Battistella e Gresse von Wangenheim, 2016a]. Esta tendência pode ser justificada pelo fato de que a maioria dos jogos educacionais é desenvolvida pelos próprios professores, com recursos e tempo muito limitados. E assim, como o desenvolvimento de jogos digitais requer um esforço considerável, muitas vezes esses jogos não alcançam todo o seu potencial de atratividade, complexidade e modo de interação [Petri et al., 2017a]. Por outro lado, jogos não-digitais tendem a ter um menor esforço no seu desenvolvimento, e parecem produzir, de forma intrínseca, um ambiente de interação social e imersão dos alunos sobre a tarefa de aprendizagem [Petri et al., 2017a; Petri et al., 2018a].

Neste contexto, diversos jogos educacionais não-digitais estão sendo desenvolvidos para potencializar o processo de aprendizagem em diferentes áreas da computação, tais como: Engenharia de Software (por exemplo, Problems and Programmers [Baker et al., 2005]), Gerenciamento de Projetos de Software (por exemplo, SCRUMIA [Gresse von Wangenheim et al., 2013b] e PlayScrum [Fernandes e Sousa, 2010]), Programação (por exemplo, C-Jump [Singh et al., 2007] e SORTIA [Battistella et al., 2017]), Segurança (por exemplo, Security Protocol Game [Hamey, 2003]), entre outros.

Deste modo, acredita-se que os jogos educacionais não-digitais potencializam diversos benefícios, como o aumento da eficácia da aprendizagem, aumento no interesse e motivação dos estudantes [Tahir e Wangmar, 2017; Çiftci, 2018]. Estes jogos podem também criar um ambiente divertido e seguro, onde os alunos podem aprender com seus próprios erros e experiências práticas em um ambiente compartilhado [Pfahl et al., 2001]. Assim, acredita-se que os jogos educacionais não-digitais possam ser uma estratégia instrucional adequada para o ensino de computação. No entanto, estas alegações são questionáveis ou não rigorosamente comprovadas, pelo fato de que a maioria das avaliações de jogos educacionais são realizadas de forma $a d-h o c$ em termos de design de pesquisa, medição, coleta e análise de dados [Calderón e Ruiz, 2015; Petri e Gresse von Wangenheim, 2017]. Assim, uma questão em aberto é se estes benefícios esperados dos jogos educacionais são, de fato, reais.

Desta forma para obter uma compreensão mais abrangente dos benefícios dos jogos educacionais não-digitais usados para o ensino de computação, foi conduzida uma série de estudos de caso de avaliação de jogos. Os estudos de caso foram conduzidos com base no modelo de avaliação de jogos educacionais MEEGA+ [Petri et al., 2017b; Petri et al., 2018b], uma evolução do modelo de avaliação de jogos mais utilizado na prática [Calderón e Ruiz, 2015; Petri e Gresse von Wangenheim, 2016; Petri e Gresse von Wangenheim, 2017]. O MEEGA+ é um modelo sistematicamente desenvolvido para a avaliação de jogos educacionais, que avalia a qualidade do jogo em termos de experiência do jogador e percepção da aprendizagem. A análise deste estudo é baseada em um conjunto de dados de 26 estudos de caso conduzidos usando o modelo MEEGA+, avaliando 9 diferentes jogos não-digitais usados para o ensino de computação em diferentes instituições de ensino do Brasil e do exterior, envolvendo uma população total de 509 alunos. 
Os resultados deste estudo podem orientar os professores na seleção de jogos não-digitais como estratégia instrucional para o ensino de computação e/ou orientar desenvolvedores de jogos em relação a aspectos a serem considerados no desenvolvimento de novos jogos não-digitais para maximizar seus benefícios aos estudantes.

\section{Método de Pesquisa}

Com o objetivo de analisar os benefícios dos jogos educacionais não-digitais usados para o ensino de computação, foi executada uma série de estudos de caso [Yin, 2017; Wohlin et al., 2012], estruturado como ilustrado na Figura 1.

\begin{tabular}{|c|c|}
\hline Estudo de caso & \multirow{3}{*}{$\begin{array}{l}\text { Métodos } \\
\text { GQM } \\
\text { [Basiliet al., 1994] }\end{array}$} \\
\hline \multirow{2}{*}{$\begin{array}{l}\text { Definição } \\
\text { Objetivo: Analisar os beneficios dos jogos educacionais não-digitais usados para o ensino de computação de modo } \\
\text { a avaliar a qualidade em termos de experiência dos jogadores e percepção da aprendizagem do ponto de vista de } \\
\text { pesquisadores no contexto de ensino de computação. }\end{array}$} & \\
\hline & \\
\hline \multicolumn{2}{|l|}{ 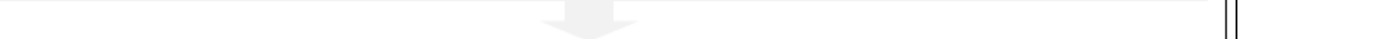 } \\
\hline $\begin{array}{l}\text { Execução } \\
\text { Etapa 1: Conduzir uma série de estudos de caso usando o modelo MEEGA+ para avaliar jogos não-digitais } \\
\text { usados para o ensino de computação. } \\
\text { Etapa 2: Coletar dados dos estudos de caso. } \\
\text { Etapa 3: Agrupar os dados coletados em uma única amostra para análise. }\end{array}$ & $\begin{array}{c}\text { Estudos de caso } \\
\text { [Yin, 2017] } \\
\text { MEEGA+ } \\
\text { [Petri et a1., 2017b] }\end{array}$ \\
\hline 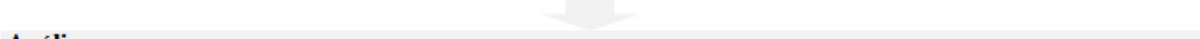 & \\
\hline $\begin{array}{l}\text { Análise } \\
\text { Questões de análise: }\end{array}$ & $\begin{array}{c}\text { GQM } \\
\text { [Basili et a1., 1994] }\end{array}$ \\
\hline $\begin{array}{l}\text { Experiência do Jogador } \\
\text { - Os jogos educacionais não-digitais proporcionam uma experiência positiva aos jogadores? }\end{array}$ & $\begin{array}{c}\text { MEEGA+ } \\
\text { [Petri et a1., 2017] }\end{array}$ \\
\hline $\begin{array}{l}\text { Percepção da Aprendizagem } \\
\text { - Os jogos educacionais não-digitais contribuem para a aprendizagem dos estudantes? }\end{array}$ & $\begin{array}{c}\text { Estatistica } \\
\text { descritiva } \\
\text { [Trochime Donnelly } \\
2008]\end{array}$ \\
\hline
\end{tabular}

Figura 1. Método de pesquisa

Seguindo a abordagem GQM (Goal/Question/Metric) [Basili et al., 1994], na fase de definição, o objetivo do trabalho é decomposto em aspectos de qualidade e questões de análise para serem analisadas com base nos dados coletados nas avaliações dos jogos. O objetivo deste estudo é analisar os benefícios dos jogos educacionais nãodigitais usados para o ensino de computação de modo a avaliar a qualidade em termos de experiência dos jogadores e percepção da aprendizagem do ponto de vista de pesquisadores no contexto de ensino de computação.

A fase de execução é organizada em três etapas. Primeiro, foram conduzidos 26 estudos de caso avaliando 9 diferentes jogos educacionais não-digitais para o ensino de computação. Os jogos usados nos estudos de caso foram selecionados de acordo com a sua adequação e relevância para o ensino de conteúdos de computação para o público alvo. Os estudos de caso foram conduzidos adotando o modelo de avaliação de jogos educacionais MEEGA+ [Petri et al., 2017b; Petri et al., 2018b]. O objetivo do modelo MEEGA+ é analisar jogos educacionais de modo a avaliar a percepção da qualidade do ponto de vista de estudantes de computação. O modelo MEEGA+ foi sistematicamente desenvolvido decompondo fatores de qualidade em um conjunto de dimensões que derivaram em um conjunto de itens que compõem um instrumento de medição para operacionalizar a coleta de dados nos estudos de caso, após a aplicação do jogo, adotando um design de pesquisa não-experimental com pós-teste. A decomposição dos fatores de qualidade do modelo MEEGA+ é apresentada na Figura 2. 


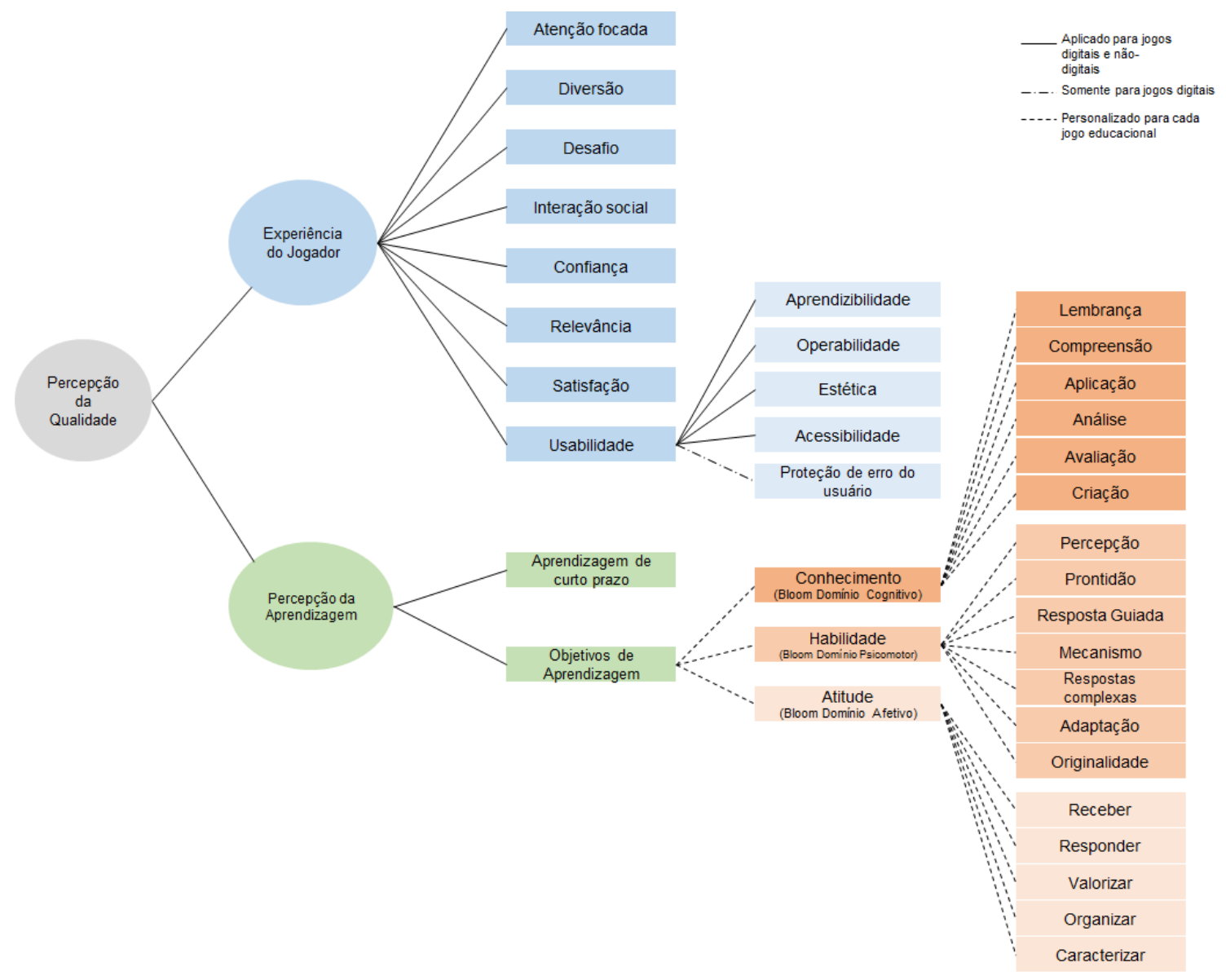

Figura 2. Decomposição dos fatores de qualidade no Modelo MEEGA+ [Petri et al., 2017b]

Cada estudo de caso para a avaliação de um jogo iniciou com a aplicação do tratamento (jogo educacional) e depois da sessão de jogo o questionário do modelo MEEGA+ foi respondido pelos estudantes de modo a coletar suas percepções (Etapa 2). Então, na etapa 3, os dados coletados em todos os estudos de caso conduzidos (Tabela 1) são agrupados em uma única amostra para a análise.

$\mathrm{Na}$ fase de análise, foi conduzida uma análise abrangente sobre os benefícios dos jogos. Os dados coletados foram analisados de forma acumulada de modo a sintetizar evidências empíricas sobre os benefícios dos jogos educacionais não-digitais em termos de experiência proporcionada as jogadores e percepção da aprendizagem. Seguindo o modelo MEEGA+ foi analisada cada uma das questões de análise definidas usando métodos de estatística descritiva. Os resultados foram interpretados e discutidos atingindo o objetivo da pesquisa.

\section{Definição e Execução do Estudo}

O objetivo deste estudo é analisar os benefícios dos jogos educacionais não-digitais usados para o ensino de computação de modo a avaliar a qualidade em termos de experiência dos jogadores e percepção da aprendizagem do ponto de vista de pesquisadores no contexto de ensino de computação. A partir deste objetivo, seguindo o modelo MEEGA+ [Petri et al., 2017b; Petri et al., 2018b], são definidas as seguintes as questões de análise (QA), agrupadas pelos fatores de qualidade avaliados: 


\section{Experiência do Jogador}

QA1: Os jogos educacionais não-digitais proporcionam uma experiência positiva aos jogadores?

\section{Percepção da Aprendizagem}

QA2: Os jogos educacionais não-digitais contribuem para a aprendizagem dos estudantes?

De modo a responder estas questões de análise, foram conduzidos 26 estudos de caso, avaliando 9 jogos educacionais não-digitais em cursos de computação usando o modelo MEEGA+. Todos os 26 estudos de caso foram conduzidos sob supervisão de ao menos um autor deste trabalho. Como resultado, obteve-se dados coletados de 509 estudantes em 4 diferentes instituições de ensino do Brasil e da Espanha, como sumarizado na Tabela 1.

\section{Tabela 1. Resumo dos estudos de caso conduzidos}

\begin{tabular}{|c|c|c|c|c|}
\hline Jogo & Contexto & Disciplina/Semestre & $\begin{array}{c}\text { Instituição/ } \\
\text { País }\end{array}$ & $\begin{array}{l}\mathbf{N}^{0} \text { de } \\
\text { alunos }\end{array}$ \\
\hline \multirow{2}{*}{$\begin{array}{l}\text { PMDome } \\
\text { [PMDome, } \\
2017]\end{array}$} & $\begin{array}{l}\text { Especialização em Gestão de Tecnologia } \\
\text { da Informação }\end{array}$ & Introdução a Gestão de TI/2016-2 & $\begin{array}{c}\text { Instituto Federal } \\
\text { Farroupilha/Brasil }\end{array}$ & 20 \\
\hline & Curso Técnico em Informática & Oficina de Jogos/2016-2 & $\begin{array}{l}\text { Instituto Federal de } \\
\text { Santa Catarina/Brasil }\end{array}$ & 27 \\
\hline \multirow{6}{*}{$\begin{array}{l}\text { Risk } \\
\text { Management } \\
\text { Game }\end{array}$} & Graduação em Ciência da Computação & Planejamento e Gestão de Projetos/2016-2 & \multirow{4}{*}{$\begin{array}{c}\text { Universidade Federal } \\
\text { de Santa } \\
\text { Catarina/Brasil }\end{array}$} & 31 \\
\hline & Graduação em Sistemas de Informação & Gestão de Projetos /2016-2 & & 23 \\
\hline & Graduação em Ciência da Computação & Planejamento e Gestão de Projetos /2017-1 & & 21 \\
\hline & Graduação em Sistemas de Informação & Gestão de Projetos /2017-1 & & 21 \\
\hline & Curso Técnico em Informática & Oficina de Jogos /2017-1 & \multirow{2}{*}{$\begin{array}{l}\text { Instituto Federal de } \\
\text { Santa Catarina/Brasil }\end{array}$} & 36 \\
\hline & Curso Técnico em Informática & Oficina de Jogos /2017-1 & & 31 \\
\hline \multirow{4}{*}{$\begin{array}{l}\text { PMMaster } \\
\text { [Gresse von } \\
\text { Wangenheim, } \\
\text { 2012] }\end{array}$} & Graduação em Ciência da Computação & Planejamento e Gestão de Projetos /2016-2 & \multirow{4}{*}{$\begin{array}{c}\text { Universidade Federal } \\
\text { de Santa } \\
\text { Catarina/Brasil }\end{array}$} & 24 \\
\hline & Graduação em Sistemas de Informação & Gestão de Projetos /2016-2 & & 21 \\
\hline & Graduação em Ciência da Computação & Planejamento e Gestão de Projetos /2017-1 & & 17 \\
\hline & Graduação em Sistemas de Informação & Gestão de Projetos /2017-1 & & 18 \\
\hline \multirow{2}{*}{$\begin{array}{l}\text { Detective Game } \\
\text { - what killed the } \\
\text { project? } \\
\text { [Gresse von } \\
\text { Wangenheim et } \\
\text { al., 2014] }\end{array}$} & \multirow[b]{2}{*}{ Graduação em Ciência da Computação } & Planejamento e Gestão de Projetos /2016-2 & \multirow[b]{2}{*}{$\begin{array}{c}\text { Universidade Federal } \\
\text { de Santa } \\
\text { Catarina/Brasil }\end{array}$} & 26 \\
\hline & & Planejamento e Gestão de Projetos /2017-1 & & 17 \\
\hline \multirow{2}{*}{$\begin{array}{l}\text { SCRUMIA } \\
\text { [Gresse von } \\
\text { Wangenheim et } \\
\text { al., 2013b] }\end{array}$} & \multirow[b]{2}{*}{ Graduação em Ciência da Computação } & Planejamento e Gestão de Projetos /2016-2 & \multirow[b]{2}{*}{$\begin{array}{c}\text { Universidade Federal } \\
\text { de Santa } \\
\text { Catarina/Brasil }\end{array}$} & 26 \\
\hline & & Planejamento e Gestão de Projetos /2017-1 & & 19 \\
\hline $\begin{array}{l}\text { Ball Point Game } \\
\text { [Gloger, 2017] }\end{array}$ & Graduação em Engenharia Informática & Workshop Jogos Educacionais/2017-2 & $\begin{array}{l}\text { Universidade de } \\
\text { Cádiz/Espanha }\end{array}$ & 10 \\
\hline $\begin{array}{l}\text { Dealing with } \\
\text { difficult people } \\
\text { [Gresse von } \\
\text { Wangenheim et } \\
\text { al., 2013a] }\end{array}$ & Graduação em Engenharia Informática & Workshop Jogos Educacionais/2017-2 & $\begin{array}{l}\text { Universidade de } \\
\text { Cádiz/Espanha }\end{array}$ & 10 \\
\hline \multirow{4}{*}{\begin{tabular}{l|} 
SORTIA - \\
Quicksort \\
Tabuleiro \\
[Battistella et \\
al., 2017]
\end{tabular}} & Graduação em Ciência da Computação & Estrutura de Dados/2016-02 & \multirow{4}{*}{$\begin{array}{c}\text { Universidade Federal } \\
\text { de Santa } \\
\text { Catarina/Brasil }\end{array}$} & 21 \\
\hline & Graduação em Sistemas de Informação & Estrutura de Dados/2016-02 & & 6 \\
\hline & Graduação em Ciência da Computação & Estrutura de Dados/2017-01 & & 25 \\
\hline & Graduação em Sistemas de Informação & Estrutura de Dados/2017-01 & & 6 \\
\hline \multirow{2}{*}{$\begin{array}{l}\text { SORTIA - } \\
\text { Heapsort }\end{array}$} & Graduação em Ciência da Computação & Estrutura de Dados/2016-02 & \multirow{2}{*}{$\begin{array}{c}\text { Universidade Federal } \\
\text { de Santa }\end{array}$} & 17 \\
\hline & Graduação em Sistemas de Informação & Estrutura de Dados/2016-02 & & 7 \\
\hline
\end{tabular}




\begin{tabular}{|l|l|l|c|c|}
\hline \begin{tabular}{l} 
Tabuleiro $\begin{array}{l}\text { [Battistella } \\
\text { al., 2017] }\end{array}$ \\
\cline { 3 - 4 }
\end{tabular} & Graduação em Ciência da Computação & Estrutura de Dados/2017-01 & Catarina/Brasil & 23 \\
\cline { 2 - 5 } & Graduação em Sistemas de Informação & Estrutura de Dados/2017-01 & 6 \\
\hline
\end{tabular}

Em síntese, os estudos de caso envolveram 9 diferentes jogos educacionais nãodigitais para o ensino de computação. A maioria dos jogos avaliados ( 7 jogos) concentram-se na área de Engenharia de Software, sendo aplicados, principalmente, em disciplinas de Planejamento e Gestão de Projetos. Estes jogos, tipicamente, são utilizados para revisar conhecimentos por meio de perguntas e respostas, como no jogo PMMaster, simular a execução de um projeto, como nos jogos SCRUMIA, PMDome e Detective Game e também, para o desenvolvimento de habilidades interpessoais, como no jogo Dealing with Difficult People. Além disso, outros dois jogos avaliados, objetivam simular na prática, em um tabuleiro, a execução dos algoritmos de ordenação Quicksort e Heapsort, em disciplinas de Estruturas de Dados. Uma visão geral de cada jogo avaliado é apresentada na Tabela 2.

Tabela 2. Visão geral dos jogos avaliados e fotos dos estudos de caso

PMDome [PMDome, 2017] é uma dinâmica de
simulação que tem como objetivo de aprendizagem
transmitir a importância do planejamento e o impacto que
a falta do mesmo pode ter. A dinâmica simula as fases de
planejamento e execução do gerenciamento de projetos,
onde os estudantes precisam planejar o tempo e os
recursos (canetas, folhas de papel e fita adesiva) que
precisarão para desenvolver o produto final, um Domo
Geodésio.

Risk Managemente Game é um jogo de tabuleiro e objetiva motivar a importância do planejamento de riscos no gerenciamento de projetos. No jogo, os jogadores precisam chegar até a entrega (final do tabuleiro), começando no planejamento e passando pelas Sprints com os recursos que the forem entregues no início da partida. No início do jogo os jogadores devem investir seus recursos em cartas de imprevistos (financeiro, material, equipe, concorrência e econômico) que especifica de onde os recursos serão tirados caso não planejado o suficiente. Cartas de Sprint determinam como os jogadores enfrentam os imprevistos na partida.

PMMaster [Gresse von Wangenheim, 2012] é um jogo de tabuleiro com perguntas sobre gerenciamento de projetos em diferentes áreas de conhecimento, como escopo, tempo e gerenciamento de qualidade. O jogador que primeiro responder corretamente uma questão de cada uma das áreas de conhecimento do PMBOK, vence o jogo. Tem como objetivo de aprendizagem revisar e reforçar os conceitos básicos de gerenciamento de projetos de acordo com o PMBOK (4 edição), focando especificamente em gestão de projetos de software.
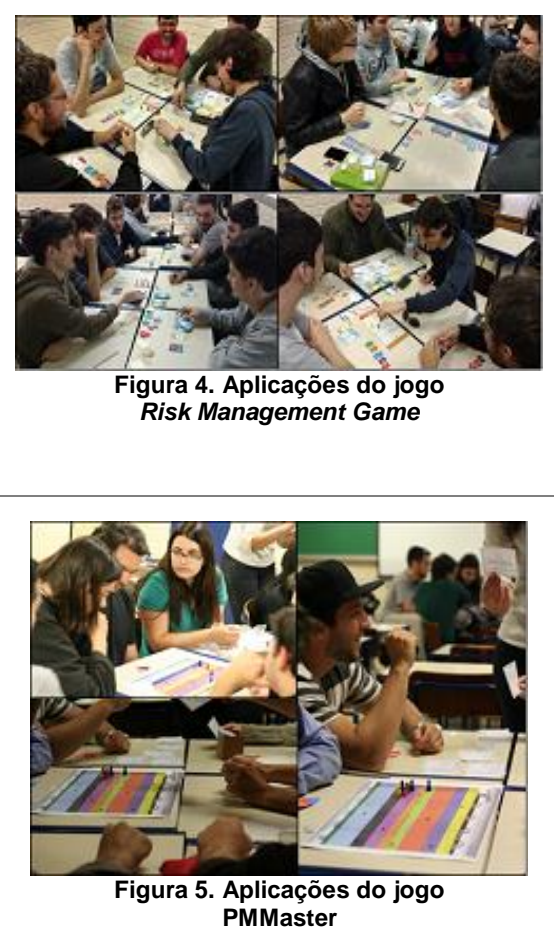
Detective Game - what killed the project? [Gresse von Wangenheim et al., 2014] é um jogo de papel \& caneta dedutivo que objetiva aplicar o Gerenciamento de Valor Agregado para o monitoramento e controle de um projeto de software como parte do ensino de gerenciamento de projetos. O objetivo de aprendizagem do jogo é reforçar os conceitos e ensinar a competência de aplicar cálculos básicos sobre o Gerenciamento de Valor Agregado.

SCRUMIA [Gresse von Wangenheim et al., 2013b] é um jogo de papel \& caneta de simulação em grupo com a finalidade de planejar e executar Sprints de um projeto hipotético aplicando SCRUM como parte de um curso de gerenciamento de projetos. O objetivo de aprendizagem da atividade é reforçar os conceitos e ensinar a competência de aplicar o conhecimento sobre o gerenciamento ágil de projetos usando SCRUM.

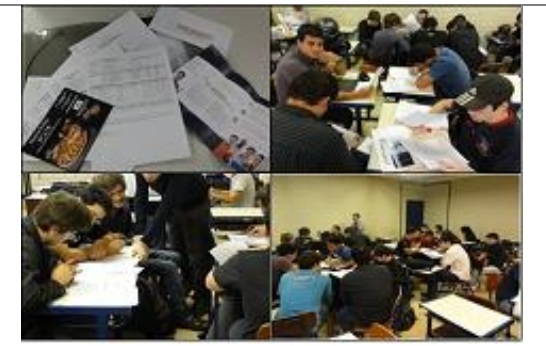

Figura 6. Aplicações do jogo

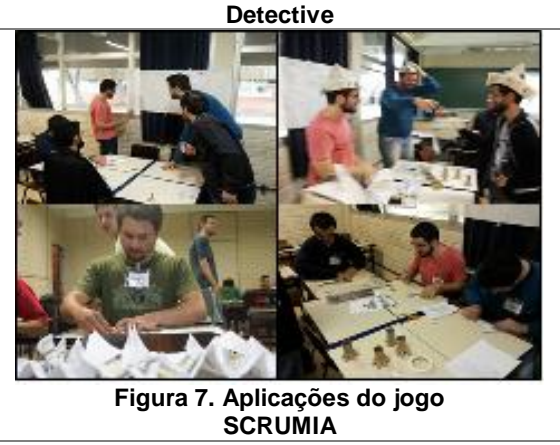

Ball Point Game [Gloger, 2017] objetiva ilustrar a dinâmica de uma equipe trabalhando iterativamente e em melhoria contínua. No jogo, organizado em iterações, as equipes devem passar tantas bolas quanto possível por todos os participantes, até retornarem ao ponto de partida, para marcar pontos. As bolas não podem ser passadas para os vizinhos à direita e à esquerda, não podem tocar o chão e as bolas devem passar pelo ar para o outro jogador. No final das iterações, a equipe que marcou mais pontos é a vencedora.

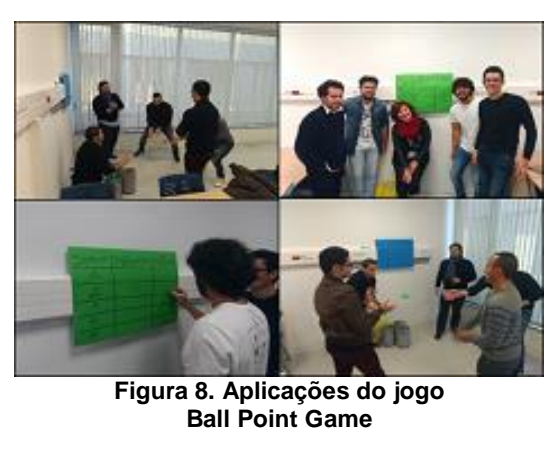

Dealing with difficult people [Gresse von Wangenheim et al., 2013a] é uma dinâmica que objetiva a ilustrar as dificuldades relacionadas à gerência de equipes em projetos de software. Os alunos em grupo simulam uma reunião de kick-off de um projeto de software. Um dos alunos assume o papel de gerente de projeto, que conduz a reunião e ao final precisa ter recebido as assinaturas de todos os membros do grupo. Cada membro do grupo recebe uma caracterização de uma personalidade difícil (p.ex. pessoa negativa, explodindo com facilidade, reclamando de tudo) e deve agir de acordo com ela até o momento em que o gerente de projeto começar a reagir

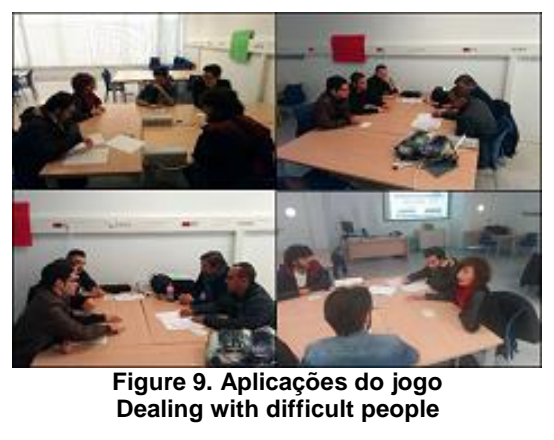
de forma adequada.

SORTIA - Quicksort e Heapsort [Battistella et al., 2017] é um jogo de tabuleiro usado para representar o modelo de memória de uma sequência de chamadas recursivas do algoritmo Quicksort e também permite simular a execução das etapas individuais do algoritmo Quicksort e do algoritmo Heapsort. O jogo é projetado para ser jogado em grupos de 4-6 alunos. No jogo, os alunos precisam ordenar 21 valores (números aleatórios de 0-100) em uma matriz, de acordo com o algoritmo Quicksort/Heapsort.

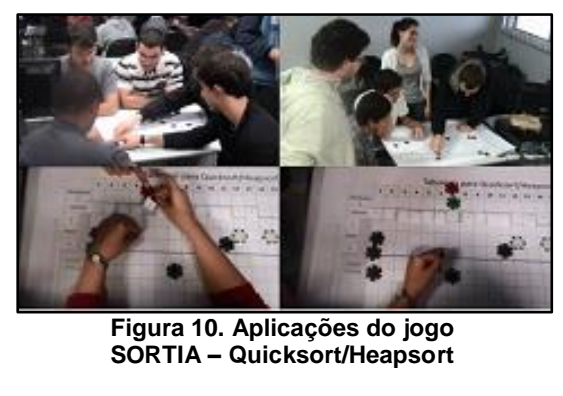


No total de 26 estudos de caso conduzidos, participaram do estudo 509 alunos, 430 homens (85\%), 76 mulheres (15\%) e 3 não responderam. A maioria dos alunos possui entre 18 e 28 anos (74\%), 18\% possuem menos de 18 anos, e 36 alunos possuem acima de 29 anos. A maioria dos participantes (56\%) indica que costuma jogar jogos não-digitais raramente, $21 \%$ jogam ao menos uma vez ao mês, $11 \%$ jogam semanalmente, $8 \%$ nunca jogaram e, 4\% dos alunos afirmam que costumam jogar diariamente.

\section{Análise dos dados}

Os dados coletados nos estudos de caso (Tabela 1) foram agrupados em uma única amostra, utilizando-os cumulativamente para analisar os benefícios dos jogos (e não para avaliar um jogo específico). Os estudos conduzidos são semelhantes em termos de definição (com o objetivo de avaliar um jogo não-digital para o ensino de computação em relação à experiência do jogador e percepção de aprendizagem), design de pesquisa (estudos de caso) e contexto (ensino de computação). Além disso, todos os estudos de caso conduzidos são padronizados em termos de medidas (fatores/dimensão de qualidade), método de coleta de dados (questionário do modelo MEEGA+) e formato de resposta (Escala Likert de 5 pontos) variando de -2 (discordo fortemente) a 2 (concordo fortemente).

Os dados coletados são analisados usando estatística descritiva em termos de distribuição de frequência e tendência central (mediana) para responder a cada uma das questões de análise definidas.

\section{QA1: Os jogos educacionais não-digitais proporcionam uma experiência positiva aos jogadores?}

De acordo com o modelo MEEGA+, a experiência proporcionada aos jogadores é avaliada em termos de usabilidade do jogo, confiança, desafio, satisfação, interação social, diversão, atenção focada e relevância. Assim, analisando os dados coletados em relação a este fator de qualidade, identificou-se que os jogos educacionais não-digitais, de forma geral, proporcionam uma experiência positiva aos estudantes de computação (Figura 11), em especial, em termos de interação social, diversão e relevância. Isso demonstra que os estudantes sentiram os jogos como uma estratégia de aprendizado positiva e engajadora.

Analisando a usabilidade dos jogos avaliados, em geral, os alunos a avaliaram de forma positiva, indicando que os jogos possuem uma boa usabilidade, que as fontes usadas nos jogos são legíveis, as cores são compreensíveis, os textos, cores e fontes combinam e são consistentes e também que as regras são claras. Em especial, os jogos PMMaster e Detective Game demostraram um alto grau de usabilidade, sendo avaliados com um design atraente e consistente. Os alunos também avaliaram de forma bastante positiva a facilidade de aprender a jogar o jogo Ball Point Game, indicando que as regras estavam claras e bastante compreensíveis, o que facilitou a iniciar a jogá-lo. 


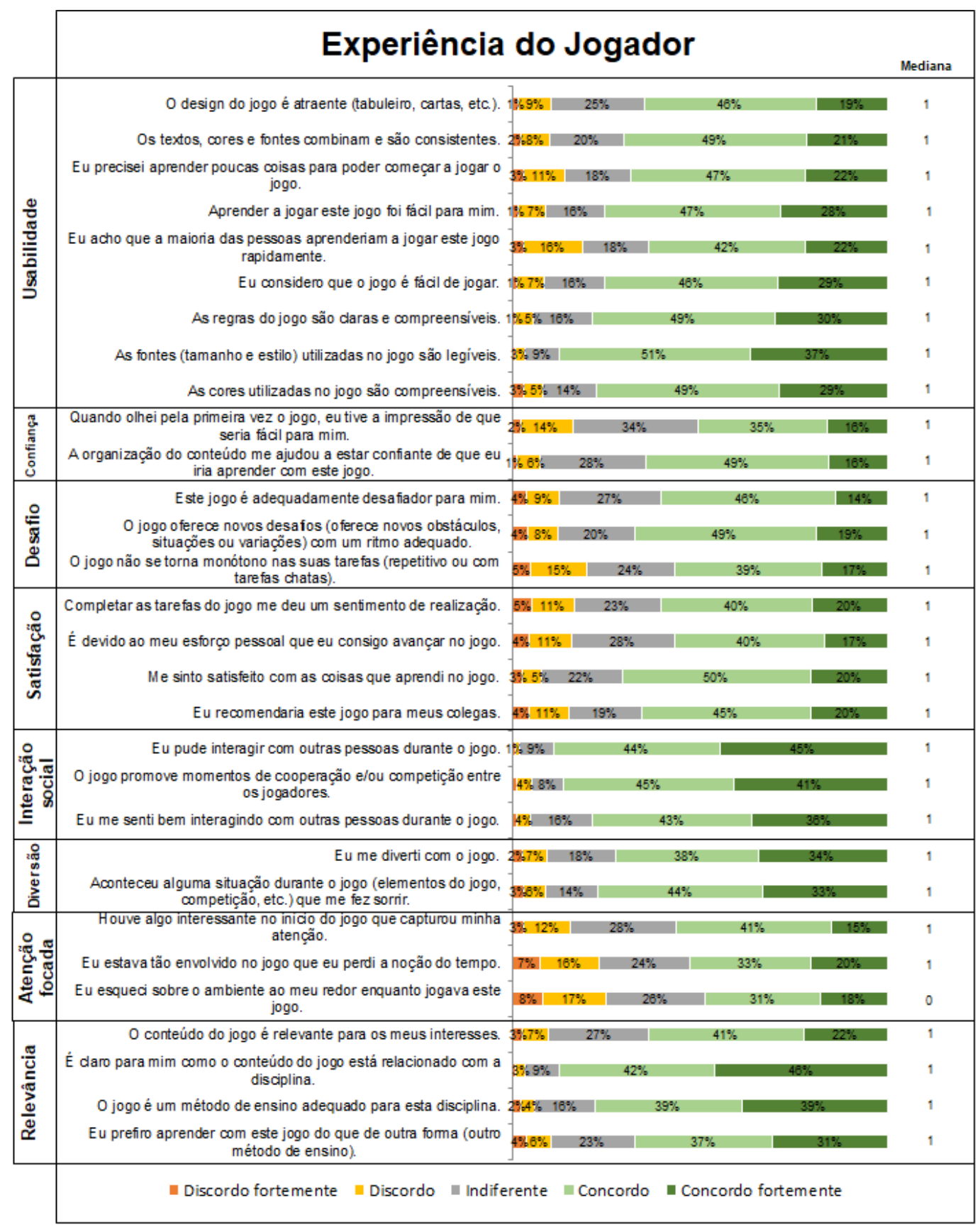

\section{Figura 11. Diagrama de frequência e medianas da avaliação da experiência do jogador baseado no total de 509 respostas}

No entanto, identificou-se que os itens relacionados a capacidade de aprender a jogar os jogos, em geral, tiveram uma avaliação mais baixa, assim, indicando que os jogos não foram tão fáceis de aprender a jogar e que possivelmente as pessoas não aprenderiam a jogar rapidamente. Um exemplo está no jogo Risk Management Game, onde os alunos devem tomar decisões iniciais de investimento de seus recursos em cartas de imprevistos (financeiro, material, equipe, concorrência e econômico) para usarem em toda partida do jogo, no entanto, o grande número de regras do jogo, pode fazer com que os alunos tomem decisões sem entender completamente o funcionamento global do jogo e, assim, o avaliaram como difícil de aprender a jogar. Outro exemplo 
está no jogo SORTIA, onde exige que os alunos saibam as etapas de execução dos algoritmos Heapsort e Quicksort, sendo indicado como difícil para as pessoas aprenderem a jogar rapidamente, pois, de fato, necessita um conhecimento prévio dos algoritmos citados. Em síntese, estes resultados, podem também estar relacionado as características deste tipo de jogos, que como são jogos não-digitais, no início da partida o instrutor deve explicar as regras e a mecânica do jogo, e assim, a forma como o instrutor as explica pode fazer com que os jogadores, em um primeiro momento, vejam o jogo com mais dificuldade, até entenderem todas as regras e começarem a jogar.

De igual modo, analisando a confiança dos jogadores ao jogar, embora avaliado de forma positiva pela maioria, alguns estudantes também indicaram que ao olhar o jogo pela primeira vez, tiveram impressão de que não seria fácil de jogar. Porém, as regras compreensíveis e a organização do conteúdo do jogo ajudaram os estudantes a estarem confiantes de que iriam aprender com o jogo.

Analisando os desafios proporcionados pelos jogos, a maioria dos estudantes os avaliou de forma positiva, indicando que os jogos são desafiadores, que os jogos vão oferecendo novos desafios aos jogadores em um ritmo adequado e que, de forma geral, os jogos não se tornam repetitivos ou com tarefas monótonas. No entanto, cabe destacar, que este último item também teve avaliações baixas, ou seja, alguns jogos foram percebidos monótonos em suas atividades. Um exemplo é o jogo PMDome, onde os alunos tinham que criar 65 tubos de papel para a montagem do Domo Geodésio, sendo avaliado como uma tarefa repetitiva pelos alunos. Deste modo, este resultado mostra que é importante avaliar o jogo para, de fato, identificar seus pontos fracos e então, melhorá-lo de modo a torná-lo mais atrativo e divertido para os alunos, minimizando as tarefas que são consideradas monótonas e/ou repetitivas pelos alunos.

Em termos de satisfação proporcionada pelos jogos, os alunos confirmaram que estão satisfeitos com os conteúdos que aprenderam jogando e recomendariam os jogos para seus colegas. Além disso, a maioria dos alunos também confirmou que conseguiram avançar no jogo devido ao seu esforço pessoal - o que corresponde a um elemento essencial de um jogo educacional, que só deve permitir que os alunos vençam se alcançaram os respectivos objetivos de aprendizagem [Abt, 2002].

A interação social foi a dimensão melhor avaliada em todos os jogos. A maioria dos estudantes concordou fortemente que os jogos promoveram momentos de cooperação e competição entre os jogadores, confirmando também que se divertiram ao interagir com outras pessoas durante o jogo. Esse comportamento pôde ser observado durante as aplicações dos jogos, o que aconteceu de forma muito agradável, proporcionando um sentimento de ambiente compartilhado. A interação social promovida pelos jogos em grupos, em vários casos, também estimulou os alunos a se familiarizarem também em ambientes fora da sala de aula.

A diversão proporcionada pelos jogos, também foi uma das dimensões melhor avaliadas pelos estudantes, confirmando que eles se divertiram jogando e que alguma situação dos jogos os fizeram sorrir. Esta característica dos jogos também é importante, pelo fato de proporcionar um ambiente mais agradável para os alunos, ao mesmo tempo que estão aprendendo enquanto estão jogando [Abt, 2002].

Analisando a atenção focada proporcionada pelos jogos, embora também avaliada de forma positiva, cabe destacar as avaliações neutras e negativas para estes itens. Os jogos não-digitais, por usarem tabuleiros, cartas e papeis, normalmente com 
um design simples, em um primeiro momento, pode não capturar a atenção dos estudantes de computação que, em geral, podem estar acostumados com os gráficos 3D de jogos digitais que costumam jogar [Battistella e Gresse von Wangenheim, 2016b]. Assim, é importante que outros fatores dos jogos (como desafio, interação social e diversão) sejam explorados para que os jogadores possam imergir na atividade de aprendizagem perdendo a noção do entorno e focando nas atividades do jogo.

A relevância dos jogos para os estudantes também foi avaliada de forma bastante positiva. Os alunos claramente perceberam que os conteúdos abordados nos jogos estão relacionados com a disciplina em que o jogo foi utilizado. A maioria dos alunos aponta que os jogos são um método de ensino adequado para a disciplina e que preferem aprender jogando, mesmo que alguns alunos indicam que prefiram aprender com outros métodos de ensino. Este resultado mostra que os alunos, apesar de estarem mais acostumados com jogos digitais, vivenciaram uma experiência positiva e relevante para a aprendizagem dos conteúdos envolvidos no jogo. Deste modo, os jogos educacionais não-digitais podem ser uma estratégia instrucional que contribui positivamente na experiência do aluno em sala de aula.

\section{QA2: Os jogos educacionais não-digitais contribuem para a aprendizagem dos estudantes?}

Analisando a percepção de aprendizagem promovida pelos jogos avaliados, em geral, pode-se observar que os jogos contribuíram para a aprendizagem dos conteúdos de computação (Figura 12). Os estudantes também indicaram que os jogos não-digitais usados para o ensino de computação foram eficientes para a sua aprendizagem, quando comparado a outras atividades realizadas na disciplina em que o jogo foi aplicado. No entanto, algumas avaliações baixas também foram identificadas. Como por exemplo, no jogo Risk Management Game, que possui como objetivo de aprendizagem motivar os alunos sobre a importância do planejamento de riscos no gerenciamento de projetos e, de fato, voltado a aprendizagem de atitude e não de conhecimento. Deste modo, este resultado pode estar indicando que alguns alunos podem preferir outros métodos de ensino.

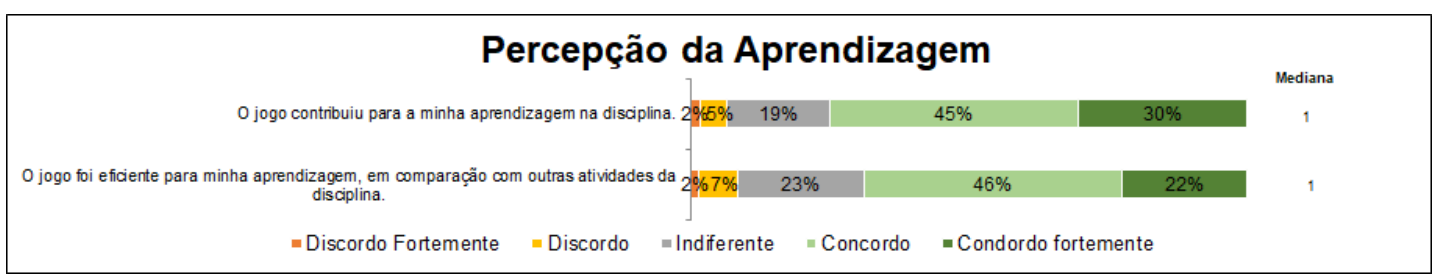

Figura 12. Diagrama de frequência e medianas da avaliação da percepção da aprendizagem

Adicionalmente, os objetivos de aprendizagem de cada jogo também foram avaliados pelos estudantes. Os resultados da avaliação de cada jogo são apresentados na Tabela 3.

Tabela 3. Resultados da avaliação dos objetivos de aprendizagem

\begin{tabular}{|l|l|c|}
\hline \multicolumn{1}{|c|}{ Jogo } & \multicolumn{1}{|c|}{ Objetivos de aprendizagem } & Mediana \\
\hline PMDome & O jogo contribuiu para reconhecer a importância da gestão (de material, equipe, tempo, riscos, etc.). & 2 \\
\hline $\begin{array}{l}\text { Risk } \\
\text { Management } \\
\text { Game }\end{array}$ & O jogo contribuiu para reconhecer a importância do planejamento de riscos. & 1 \\
\hline PMMaster & O jogo contribuiu para reforçar e fixar os conceitos sobre os grupos de processos de gerenciamento de & 1 \\
\hline
\end{tabular}




\begin{tabular}{|c|c|c|}
\hline & projetos (iniciação, planejamento, etc.). & \\
\hline & $\begin{array}{l}\text { O jogo contribuiu para reforçar e fixar os conceitos sobre as áreas de conhecimento de gerenciamento } \\
\text { de projetos (escopo, tempo, custo, etc.). }\end{array}$ & 1 \\
\hline \multirow{3}{*}{$\begin{array}{l}\text { Detective } \\
\text { Game }\end{array}$} & O jogo contribuiu para compreender a técnica de valor agregado. & 1 \\
\hline & O jogo contribuiu para aplicar na prática a técnica de valor agregado. & 1 \\
\hline & O jogo contribuiu para compreender os processos de monitoramento e controle. & 1 \\
\hline \multirow{12}{*}{ SCRUMIA } & O jogo contribuiu para relembrar os conceitos sobre o Planejamento de uma Sprint. & 1 \\
\hline & O jogo contribuiu para relembrar os conceitos sobre o Monitoramento de uma Sprint. & 1 \\
\hline & O jogo contribuiu para relembrar os conceitos sobre a Reunião de revisão de uma Sprint. & 1 \\
\hline & O jogo contribuiu para relembrar o que é o Taskboard. & 1 \\
\hline & $\begin{array}{l}\text { O jogo contribuiu para diferenciar os papéis, reuniões e artefatos envolvidos no Planejamento de uma } \\
\text { Sprint. }\end{array}$ & 1 \\
\hline & $\begin{array}{l}\text { O jogo contribuiu para diferenciar os papéis, reuniões e artefatos envolvidos no Monitoramento de uma } \\
\text { Sprint. }\end{array}$ & 1 \\
\hline & $\begin{array}{l}\text { O jogo contribuiu para diferenciar os papéis, reuniões e artefatos envolvidos na Reunião de revisão de } \\
\text { uma Sprint. }\end{array}$ & 1 \\
\hline & O jogo contribuiu para diferenciar a estrutura de organização do Taskboard. & 1 \\
\hline & O jogo contribuiu para praticar o Planejamento de uma Sprint. & 1 \\
\hline & O jogo contribuiu para praticar o Monitoramento de uma Sprint. & 1 \\
\hline & O jogo contribuiu para praticar a Reunião de revisão de uma Sprint. & 1 \\
\hline & O jogo contribuiu para praticar a organização de um Taskboard. & 1 \\
\hline \multirow{4}{*}{$\begin{array}{l}\text { Ball Point } \\
\text { Game }\end{array}$} & O jogo contribuiu para perceber a importância do trabalho em equipe para alcançar os objetivos. & 2 \\
\hline & O jogo contribuiu para perceber a importância da re (organização) em uma equipe de trabalho. & 2 \\
\hline & O jogo contribuiu para perceber a importância da comunicação em uma equipe de trabalho. & 2 \\
\hline & O jogo contribuiu para perceber a importância da avaliação do trabalho para sua melhora contínua. & 2 \\
\hline \multirow{4}{*}{$\begin{array}{l}\text { Dealing with } \\
\text { difficult } \\
\text { people }\end{array}$} & $\begin{array}{l}\text { O jogo contribuiu para perceber as dificuldades colocadas pelas diferentes personalidades que podem } \\
\text { existir em uma equipe de trabalho. }\end{array}$ & 2 \\
\hline & $\begin{array}{l}\text { O jogo contribuiu para perceber como a personalidade dos membros de uma equipe influencia na } \\
\text { produtividade da equipe de trabalho. }\end{array}$ & 2 \\
\hline & $\begin{array}{l}\text { O jogo contribuiu para perceber a importância de saber gerenciar as diferentes personalidades dos } \\
\text { membros em uma equipe de trabalho. }\end{array}$ & 1 \\
\hline & $\begin{array}{l}\text { O jogo contribuiu para perceber a importância da comunicação dentro de uma equipe de trabalho na } \\
\text { hora de gerar ideias. }\end{array}$ & 2 \\
\hline \multirow{3}{*}{$\begin{array}{l}\text { SORTIA - } \\
\text { Quicksort } \\
\text { Tabuleiro }\end{array}$} & O jogo contribuiu para reforçar os conhecimentos sobre o Algoritmo Quicksort. & 1 \\
\hline & O jogo contribuiu para compreender o Algoritmo Quicksort. & 1 \\
\hline & O jogo contribuiu para aplicar na prática os conhecimentos sobre o Algoritmo Quicksort. & 1 \\
\hline \multirow{3}{*}{$\begin{array}{l}\text { SORTIA - } \\
\text { Heapsort } \\
\text { Tabuleiro }\end{array}$} & O jogo contribuiu para reforçar os conhecimentos sobre o Algoritmo Heapsort. & 2 \\
\hline & O jogo contribuiu para compreender o Algoritmo Heapsort. & 2 \\
\hline & O jogo contribuiu para aplicar na prática os conhecimentos sobre o Algoritmo Heapsort. & 2 \\
\hline
\end{tabular}

Analisando os resultados dos objetivos de aprendizagem, pode-se observar que, de forma geral, todos os 9 jogos educacionais não-digitais aplicados foram avaliados de forma bastante positiva, indicando assim, uma percepção por parte dos alunos que os jogos alcançaram seus objetivos de aprendizagem. No entanto, observa-se uma avaliação levemente mais positiva referente aos jogos PMDome, Ball Point Game e Dealing with Defficult People. Estes jogos possuem como objetivo de aprendizagem motivar a importância do planejamento e trabalhar habilidades de trabalho em grupo, comunicação e gestão de equipes, assim, podem ter sido avaliados de forma mais positiva pelos alunos pelo fato de desenvolver, em forma de jogo, habilidades importantes para os estudantes de computação. De forma similar, os alunos também concordaram fortemente que o jogo SORTIA-Heapsort alcançou os objetivos de aprendizagem de reforçar, compreender e aplicar na prática os conhecimentos sobre o algoritmo Heapsort. Este resultado, quando comparado ao resultado do jogo SORTIAQuicksort, pode estar relacionado aos conhecimentos prévios dos alunos sobre o 
algoritmo Heapsort, necessários para a execução do jogo. Em síntese, os jogos nãodigitais, além de serem um método de ensino que proporciona uma experiência positiva aos alunos, também contribui para a aprendizagem dos estudantes de computação.

\subsection{Ameaças à validade}

Devido às características deste tipo de pesquisa, este trabalho está sujeito a ameaças à validade. Deste modo, foram identificadas ameaças potenciais e aplicadas estratégias de mitigação para minimizar o impacto no trabalho.

Algumas ameaças estão relacionadas ao design de pesquisa [Wohin et al., 2012]. Considerando o contexto deste estudo, relacionado a ensino de computação, isso significa que deve ser possível realizar a avaliação do jogo de forma rápida e não intrusiva, de modo a não interromper o fluxo normal das aulas. Portanto, optou-se por conduzir uma série de estudos de caso de avaliação que permitem realizar uma pesquisa aprofundada de um indivíduo, grupo ou evento (Yin, 2014; Wohlin et al., 2012). Experimentos, por outro lado, causariam uma interrupção maior nas aulas, além de exigir a definição de grupos de controle que podem ser prejudicados ao usar métodos de ensino alternativos considerados inferiores. Além disso, para obter resultados estatisticamente significativos de tais experimentos, é necessário um tamanho de amostra considerável, o que dificultaria a condução devido ao número reduzido de alunos comumente matriculados em disciplinas de computação.

Outro risco se refere a confiabilidade e validade dos dados coletados nos estudos de caso de avaliação. Para minimizar este risco, todos os estudos de caso foram conduzidos adotando o modelo MEEGA+ [Petri et al., 2017b; Petri et al., 2018b]. O MEEGA+ é um modelo sistematicamente desenvolvido para a avaliação de jogos educacionais e amplamente avaliado em termos de validade e confiabilidade [Petri et al., 2018b].

Outra ameaça está na avaliação da aprendizagem, como não houve um pré-teste medindo, por exemplo, o nível de conhecimento dos alunos antes da aplicação dos jogos, não foi possível identificar com exatidão o quanto cada aluno aprendeu sobre os conteúdos abordados nos jogos. No entanto, embora sem consenso, existem evidências de que a auto avaliação fornece informações confiáveis, válidas e úteis para este tipo de estudo [Sitzmann et al., 2010].

Em termos de validade externa, uma ameaça à possibilidade de generalizar os resultados está relacionada ao tamanho da amostra e à diversidade dos dados utilizados para a avaliação. Com relação ao tamanho da amostra, a avaliação usou dados coletados de 26 estudos de caso que avaliaram 9 diferentes jogos não-digitais, envolvendo uma população de 509 alunos. Em termos de significância estatística, este é um tamanho de amostra satisfatório que permite a geração de resultados significativos [Sitzmann et al., 2010]. Os dados foram obtidos a partir de aplicações de jogos em 4 diferentes instituições de ensino. No entanto, como a coleta de dados foi restrita às avaliações de jogos não-digitais para o ensino de computação avaliados com o modelo MEEGA+, a maioria dos dados é do Brasil, onde é usado de forma mais proeminente, com apenas duas aplicações conduzidas pelos autores em uma universidade da Espanha.

Em termos de confiabilidade, uma ameaça refere-se a que medida os dados e a análise dependem dos pesquisadores específicos. Para mitigar essa ameaça, foi 
documentada uma metodologia de pesquisa sistemática, definindo claramente o objetivo do estudo, o processo de coleta e análise de dados.

\section{5 . Conclusão}

De modo a obter uma compreensão mais abrangente sobre os benefícios dos jogos educacionais não-digitais usados para o ensino de computação, uma série de estudos de caso aplicando e avaliando jogos não-digitais foi conduzida. Os resultados da análise de 26 estudos de caso, envolvendo 509 alunos e avaliando 9 diferentes jogos para o ensino de computação, fornecem evidências sobre os benefícios dos jogos educacionais nãodigitais que podem produzir um efeito positivo na aprendizagem dos estudantes de computação, proporcionando uma experiência agradável e envolvente aos alunos e motivando-os ao estudo.

Deste modo, pode-se concluir que os jogos educacionais não-digitais podem ser uma alternativa efetiva para o ensino de computação, quando comparado ao custo/benefício e esforço despendido para o desenvolvimento de jogos digitais [Petri et al., 2017]. Principalmente por facilmente possibilitar uma interação social entre os alunos, promovendo um ambiente agradável e divertido para a aprendizagem, estimulando a cooperação e compartilhamento de ideias entre os estudantes. Adicionalmente, os resultados também apontaram que para ter estes benefícios positivos é importante desenvolver jogos com tarefas que não sejam monótonas e/ou repetitivas, mas sim, que proporcionam desafios, diversão, competitividade e cooperação entre os jogadores.

Como trabalhos futuros pretende-se continuar a pesquisa de avaliação de jogos não-digitais em diferentes contextos, de forma a confirmar os resultados deste estudo e também identificar novas oportunidades neste tipo de jogos de modo a contribuir para sua efetiva e contínua melhoria.

\section{Agradecimentos}

Gostaríamos de agradecer aos alunos de todas as instituições de ensino que participaram das aplicações dos jogos.

Este trabalho foi realizado no âmbito do Programa de Doutorado Sanduíche no Exterior (PDSE) na Universidade de Cádiz, Espanha, patrocinado pela CAPES (Coordenação de Aperfeiçoamento de Pessoal de Nível Superior), fundação do Ministério da Educação, Brasil (nº 88881.131485 / 2016- 01).

Este trabalho foi apoiado pelo CNPq (Conselho Nacional de Desenvolvimento Científico e Tecnológico), uma entidade do governo brasileiro focada no desenvolvimento científico e tecnológico.

Este trabalho também foi apoiado pela Agencia Española de Investigación (AEI) com fundos FEDER no âmbito do projeto BadgePeople (TIN2016-76956-C3-3-R) e do Plano Andaluz de Pesquisa, Desenvolvimento e Inovação (TIC-195).

\section{Referências}

Abt, C. C. (2002). Serious Games. Lanhan: University Press of America. 
Baker, A., Navarro, E. O, e van der Hoek, A. (2005). An experimental card game for teaching software engineering processes. Journal of Systems and Software, 75(1-2), 3-16.

Basili, V. R., Caldiera, G., e Rombach, H. D. (1994). Goal, Question Metric Paradigm. In J. J. Marciniak, Encyclopedia of Software Engineering. New York, USA.

Battistella, P. E. e Gresse von Wangenheim, C. (2016a). Games for teaching computing in higher education - A systematic review. IEEE Technology and Engineering Education, 9(1), 8-30.

Battistella, P. E. e Gresse von Wangenheim, C. (2016b). Caracterização do PúblicoAlvo de Jogos Educacionais na área da Computação. $24^{\circ}$ Workshop sobre Educação em Computação, (pp. 2016-2025). Porto Alegre, RS, Brasil.

Battistella, P. E., Gresse von Wangenheim, C., von Wangenheim, A., e Martina, J. E. (2017). Design and Large-scale Evaluation of Educational Games for Teaching Sorting Algorithms. Informatics in Education, 17(2), 141-164.

Calderón A. e Ruiz M. (2015). A systematic literature review on serious games evaluation: An application to software project management. Computers \& Education, $87,396-422$.

Çiftci, S. (2018). Trends of Serious Games Research from 2007 to 2017: A Bibliometric Analysis. Journal of Education and Training Studies, 6(2), 18-27.

Fernandes, J. M., Sousa, S. M. (2010). PlayScrum - A Card Game to Learn the Scrum Agile Method. International Conference on Games and Virtual Worlds for Serious Applications, (pp.52-59). Braga, Portugal.

Gloger, B. (2017). Ball Point Game. Disponível em: <https://borisgloger.com/wpcotent/uploads/2016/08/Ball_Point_Game.pdf $>$ Acesso em: 27 novembro 2017.

Gresse von Wangenheim, C. (2012). PMMaster. Disponível em: $<$ http://www.gqs.ufsc.br/pm-master/> Acesso em: 06 fevereiro 2018.

Gresse von Wangenheim, C., Carvalho, O. P., e Battistella, P. E. (2013a) Ensinar a Gerência de Equipes em Disciplinas de Gerência de Projetos de Software. Revista Brasileira de Informática na Educação, 21(1), 15-22.

Gresse von Wangenheim, C., Rausis, B. Soares, G., Savi, R., e Borgatto, A. F. (2014). Project Detective A Game for Teaching Earned Value Management. International Journal of Teaching and Case Studies, 5(3/4), 216-234.

Gresse von Wangenheim, C., Savi, R., e Borgatto, A. F. (2013b). SCRUMIA - An educational game for teaching SCRUM in computing courses. Journal of Systems and Software, 86(10), 2675-2687.

Hamey, L. G. C. (2003). Using the Security Protocol Game to teach computer network security. Symposium on the Improving Learning Outcomes Through Flexible Science Teaching, (pp. 96-101). Sydney, Australia.

Petri, G. e Gresse von Wangenheim, C. (2016). How to Evaluate Educational Games: a Systematic Literature Review. Journal of Universal Computer Science, 22(7), 992 1021. 
Petri, G. e Gresse von Wangenheim, C. (2017). How games for computing education are evaluated: a systematic literature review. Computers \& Education, vol. 107, 6890 .

Petri, G., Gresse von Wangenheim, C., e Borgatto, A. F. (2017a). Quality of Games for Teaching Software Engineering: An Analysis of Empirical Evidences of Digital and Non-digital Games. Proc. of the 39th Int. Conf. on Software Engineering: Software Engineering Education and Training Track, (pp. 150-159). Buenos Aires, Argentina.

Petri, G., Gresse von Wangenheim, C., e Borgatto, A. F. (2017b). Evolução de um Modelo de Avaliação de Jogos para o Ensino de Computação. $25^{\circ}$ Workshop sobre Educação em Computação, (pp. 2327-2336). São Paulo, SP, Brasil.

Petri, G., Gresse von Wangenheim, C., Borgatto, A. F., Calderón, A., e Ruiz, M. (2018a). Digital Games for Computing Education: What are the Benefits? In Krassmann, A. L. et al. (Eds). Handbook of Research on Immersive Digital Games in Educational Environments. IGI Global, cap. 2 (aceito para publicação).

Petri, G., Gresse von Wangenheim, C., e Borgatto, A. F. (2018b). MEEGA+, Systematic Model to Evaluate Educational Games. In Newton Lee (Eds) Encyclopedia of Computer Graphics and Games, (pp. 1-7). Cham: Springer.

Pfahl, D., Ruhe, G., e Koval, N. (2001). An experiment for evaluating the effectiveness of using a system dynamics simulation model in software project management education. International Symposium on Software Metrics, (pp.97-109). London, GB.

PMDome. (2017). PMDome Workshop. Disponível em: $<$ https://ricardovargas.com/pt/workshops/pmdome/> Acesso em: 27 novembro 2017.

Singh, J., Dorairaj, S. K., e Woods, P. (2007). Learning computer programming using a board game - case study on C-Jump. Symposium on Information and Communications Technologies. Kuala Lumpur, Malaysia.

Sitzmann, T., Ely, K., Brown, K. G., e Bauer, K. N. (2010). Self-Assessment of Knowledge: A Cognitive Learning or Affective Measure? Academy of Management Learning \& Education, 9(2), 169-191.

Souza, M. e França, C. (2016). O que explica o sucesso de jogos no ensino de engenharia de software? Uma teoria de motivação. $24^{\circ}$ Workshop sobre Educação em Computação, (pp. 2255-2263). Porto Alegre, RS, Brasil.

Tahir, R. e Wangmar, A. I. (2017). State of the art in Game Based Learning: Dimensions for Evaluating Educational Games. Proc. of the European Conference on Games Based Learning, (pp. 641-650). Graz, Austria.

Trochim, W. M. e Donnelly, J. P. (2008). Research methods knowledge base (3 ed.). Mason: Atomic Dog Publishing.

Wohlin, C., Runeson, P., Höst, M., Ohlsson, M. C., Regnell, B., eWesslén, A. (2012). Experimentation in Software Engineering. Springer-Verlag Berlin Heidelberg.

Yin, R. K. (2017). Case study research and applications: design and methods (5 ed.). Beverly Hills: Sage Publications. 